\title{
Polarization correlometry of nets for characteristic states in Mueller-matrix patterns inherent to phase-inhomogeneous biological layers
}

\author{
O.Yu. Novakovs'ka \\ Chernivtsi National University named after Yuri Fed'kovych, \\ Department for Optics and Spectroscopy, \\ 2 Kotsyubyns 'kogo str., 58001 Chernivtsi, Ukraine. \\ Phone: 0677830572
}

\begin{abstract}
Considered in this paper are the capabilities of the singular approach to the analysis of Stokes-parametric and Mueller-matrix patterns inherent to polycrystalline networks observed in phase-inhomogeneous biological layers. Offered is the model of nets for characteristic values of Mueller matrix elements that characterize mechanisms of formation of polarization singularities in laser images. The authors have grounded application of the method of cross-correlation two-point analysis aimed at polarization images formed by linear birefringence of biological crystal networks.
\end{abstract}

Keywords: polarization, singularity, Mueller matrix, Stokes vector, optical anisotropy, biological layer, cross-correlation function.

Manuscript received 23.02.12; revised version received 17.05.12; accepted for publication 14.06.12; published online 25.09.12.

\section{Introduction}

By convention, processes of transformation of optical radiation in phase-inhomogeneous objects and substances are considered in statistical approximation. Among the most widely spread methods, one can separate the so-called "scalar" (photometry, spectrophotometry) and "vector" ones (Mueller-matrix optics). Being based on them, ascertained in the works by Wang[1], Tuchin [2], Angel'sky [3] were interrelations between the set of statistical moments of the first to fourth orders that characterize the light scattering in phase-inhomogeneous layers and distribution of polarization states in laser images. In parallel with conventional statistical investigations, formed in the works by Nye [4], Berry [5], Soskin [6] during the recent $10-15$ years is a new optical direction in describing the structure of polarizationinhomogeneous fields within the frames of the topological approach - singular optics. The new polarization-correlation approach to the analysis of spatial-coordinate distributions of coherent radiation fields offered and developed in the works by Gori and
Friberg [7, 8], Ellis and Dogariu [9] became generalization of the above directions. This, predominantly theoretical, direction of coherent optics is based on using the "two-point" parameters for description of correlation interrelations between phase shifts and polarization-singular states of various points in laser fields. Demonstrated in the works by Ushenko [...] is the capability to use these parameters in description of optical anisotropy observed in birefringent networks of biological tissues. At the same time, polarization correlometry needs further development. In the first place, to extend functional capabilities for diagnostics of optical anisotropy observed in biological layers, it seems topical to provide synthesis of both statistical (one-point) and correlation (two-point) directions when analyzing the topological structure of laser radiation fields transformed by networks of biological crystals.

Our work is aimed at search of new informative singular and polarization-correlation diagnostic parameters to characterize laser fields and polycrystalline networks of biological layers by using methods of laser polarimetry and developing the 
methods for singular and correlation reproduction of the structure inherent to networks of protein crystals.

\section{Polarization inhomogeneity and singularities in images of polycrystalline biological networks}

As a base for analyzing the mechanisms of formation of polarization-singular structure of laser fields transformed by biological tissues, we chose the well-known model approach [3], in accord with which these objects can be represented as a two-component amorphous-andcrystallite matrix. Polarization-active components in this structure are birefringent optically single-axis fibrils of biological tissues, properties of which in each point with the coordinate $(r)$ can be described with the Jones operator

$\{C\}=\left\|\begin{array}{ll}\cos ^{2} \rho+\sin ^{2} \rho \exp (-i \delta) ; & \cos \rho \sin \rho[1-\exp (-i \delta)] ; \\ \cos \rho \sin \rho[1-\exp (-i \delta)] ; & \sin ^{2} \rho+\cos ^{2} \rho \exp (-i \delta) ;\end{array}\right\|$,

where $\rho$ is the direction of folding for birefringent fibrils within the plane of the sample of biological tissue (BT), substance of which brings the phase shift $\delta$ between orthogonal components $E_{0 x}, E_{0 y}$ of the laser beam.

For simplicity (but without any loss in completeness of our analysis), let us consider the mechanisms of formation of polarization structure in images of BT when probing it with the linearly polarized laser beam possessing the azimuth $\alpha=0{ }^{0}$ relatively to the plane of incidence

$\left(\begin{array}{c}E_{0 x} \\ E_{0 y}\end{array}\right) \Rightarrow\left(\begin{array}{c}E_{0 x} \\ 0\end{array}\right)$.

With account of (2), the relations (1) can be rewritten in the following form

$$
\begin{aligned}
& E_{x}(r)=E_{0 x}\left[\cos ^{2} \rho(r)+\sin ^{2} \rho(r) \exp (-i \delta(r))\right] ; \\
& E_{y}(r)=E_{0 x}[\cos \rho(r) \sin \rho(r)(1-\exp (-i \delta(r)))] .
\end{aligned}
$$

To determine the local states of light oscillation polarization in points of the image obtained for biological tissue, let us write the respective coherency matrixes

$$
\{\mathrm{K}(r)\}=\| \begin{array}{cc}
E_{x}(r) E^{*}{ }_{x}(r) ; & E_{x}(r) E_{y}^{*}(r) ; \| \\
E_{x}^{*}(r) E_{x}(r) ; & E_{y}(r) E_{y}^{*}(r) . \| .
\end{array} .
$$

In a general case, for an elliptically polarized beam $E_{0 x}+E_{o y} \exp \left(-i \delta_{0}\right)$, the expressions describing the orthogonal amplitudes of light oscillations in the points of images obtained from optically anisotropic parts of BT take the look

$$
\begin{aligned}
& E_{x}=\cos \rho\left(E_{0 x} \cos \rho+E_{0 y} \sin \rho \exp \left(-i \delta_{0}\right)\right)+ \\
& +\sin \rho\left(E_{0 x} \sin \rho-E_{0 y} \cos \rho \exp \left(-i \delta_{0}\right)\right) \exp (-i \delta) ; \\
& E_{y}=\sin \rho\left(E_{0 x} \sin \rho+E_{0 y} \cos \rho \exp \left(-i \delta_{0}\right)\right)+ \\
& +\cos \rho\left(E_{0 x} \cos \rho-E_{0 y} \sin \rho \exp \left(-i \delta_{0}\right)\right) \exp (-i \delta) .
\end{aligned}
$$

As it follows from (5), the most probable polarization state in image points for BT is the elliptic one. It can be shown that the conditions for formation of linear polarization singularities (L-states or L-points) are defined with the following relations

$$
\sin ^{2} \rho \cos ^{2} \rho\left(E_{0 x}^{2} \cos ^{2} \rho-E_{0 y}^{2} \sin ^{2} \rho \exp \left(-2 i \delta_{0}\right)\right) \times
$$

$\times \sin ^{2} \delta=0$

$$
\rho=\operatorname{arctg}\left[\exp \left(-2 i \delta_{0}\right) \frac{E_{0 y}}{E_{0 x}}\right] \text {; }
$$$$
\delta=2 q \pi, q= \pm 0,1,2 \ldots
$$

In the case of circular polarization singularities (Cstates or C-points), the following relation is valid $\sin ^{2} \rho \cos ^{2} \rho\left(E_{0 x}^{2} \cos ^{2} \rho-E_{0 y}^{2} \sin ^{2} \rho \exp \left(-2 i \delta_{0}\right)\right) \times$ $\times \sin ^{2} \delta=1$,

which enables to determine the conditions for formation of circularly polarized point in the images of BT

$\rho=\pi / 4+\operatorname{arctg}\left[\exp \left(-2 i \delta_{0}\right) \frac{E_{0 y}}{E_{0 x}}\right] ;$.

$\delta=\pi / 2+2 q \pi, q= \pm 0,1,2,3 \ldots$

Thus, the performed analysis enables to find the conditions and possibilities for BT to "generate" the networks of polarization-singular points and to describe the main mechanisms of their appearance.

\section{Characteristic values of vector-parametric and Mueller-matrix patterns corresponding to polycrystalline networks of biological crystals}

Our analysis of the relations (1) to (9) shows that the major role in transformation of the laser radiation polarization state is played by the network of birefringent fibrils. Among the diversity of $\rho$ and $\delta$ values, there exist definite particular (characteristic) values of orientation $\rho^{*}$ and phase $\delta^{*}$ parameters inherent to this polycrystalline network

$$
\left\{\begin{array}{l}
\rho^{*}=0^{0} ; \quad \pm 45^{\circ} ; \quad 90^{\circ} ; \\
\delta^{*}=0^{\circ}, \pm 90^{\circ}, 180^{\circ} .
\end{array}\right.
$$

These polarization-singular states of laser image points are corresponded by definite extreme or characteristic values of the fourth Stokes-vector parameter

$$
S_{4}=\sin 2 \beta=\left\{\begin{array}{l}
S_{4}\left(\delta^{*}=0\right)=0 \Leftrightarrow L-\text { point; } \\
S_{4}\left(\delta^{*}=0.5 \pi\right)=+1,0 \Leftrightarrow+C-\text { point; } \\
S_{4}\left(\delta^{*}=-0.5 \pi\right)=-1,0 \Leftrightarrow-C-\text { point } .
\end{array}\right.
$$

On the other hand, the values (11) of parameters inherent to the network of biological crystals correspond to definite (characteristic) values of the elements in the Mueller matrix 


$$
\{M\}=\left\|\begin{array}{|cccc}
1 & 0 & 0 & 0 \\
0 & M_{22} & M_{23} & M_{24} \\
0 & M_{32} & M_{33} & M_{34} \\
0 & M_{42} & M_{43} & M_{44}
\end{array}\right\|=\left\|\begin{array}{cccc}
1 & 0 & 0 & 0 \\
0 & \left(\cos ^{2} 2 \rho+\sin ^{2} 2 \rho \cdot \cos \delta\right) ; & \cos 2 \rho \sin 2 \rho(1-\cos \delta) ; & (\sin 2 \rho \sin \delta) ; \\
0 & (\cos 2 \rho \sin 2 \rho(1-\cos \delta)) ; & \left(\sin ^{2} 2 \rho+\cos ^{2} 2 \rho \cos \delta\right) ; & (\cos 2 \rho \sin \delta) ; \\
0 & (-\sin 2 \rho \sin \delta) ; & (-\cos 2 \rho \sin \delta) ; & (\cos \delta) .
\end{array}\right\|
$$

In more detail, this interrelation between singular values of the set for elements in the Mueller matrix and polarization singularities in BT images is demonstrated with Table 1.

Table 1. Interrelation of characteristic values of elements in the Mueller matrix and polarizationsingular states.

\begin{tabular}{|c|c|c|c|}
\hline \multicolumn{2}{|c|}{$m_{i k}$} & $\begin{array}{c}\text { “ } L ”-\text { point } \\
(\varphi=0, \varphi=\pi)\end{array}$ & $\begin{array}{c}\text { “ } \pm C \text { "-point } \\
(\varphi= \pm \pi / 2)\end{array}$ \\
\hline \multirow{3}{*}{$m_{22}$} & 0 & - & $\gamma=\pi / 4$ \\
\cline { 2 - 4 } & 1 & $\varphi=0$ & - \\
\cline { 2 - 4 } & -1 & $\varphi=\pi$ & - \\
\hline \multirow{4}{*}{$m_{24}=-m_{42}$} & 0 & $\gamma=\pi / 2$ & - \\
\cline { 2 - 4 } & 1 & - & $\gamma= \pm \pi / 4$ \\
\cline { 2 - 4 } & -1 & - & $\gamma=\mp \pi / 4$ \\
\hline \multirow{3}{*}{$m_{44}$} & 0 & - & $\gamma=0 \div \pi$ \\
\cline { 2 - 4 } & 1 & $\varphi=0$ & - \\
\cline { 2 - 4 } & -1 & $\varphi=\pi$ & - \\
\hline
\end{tabular}

Having determined the coordinate distribution of characteristic values from the Mueller matrix, we predicted and grounded the interrelation between networks of polarization singularities and distributions of characteristic values for vector-parametric and Mueller-matrix patterns.

The structure of characteristic values for Stokesparametric pattern $V_{4}(x, y)$ corresponding to histological sections of the most spread types of BT - connective tissue (skin derma) is depicted in Fig. 1.

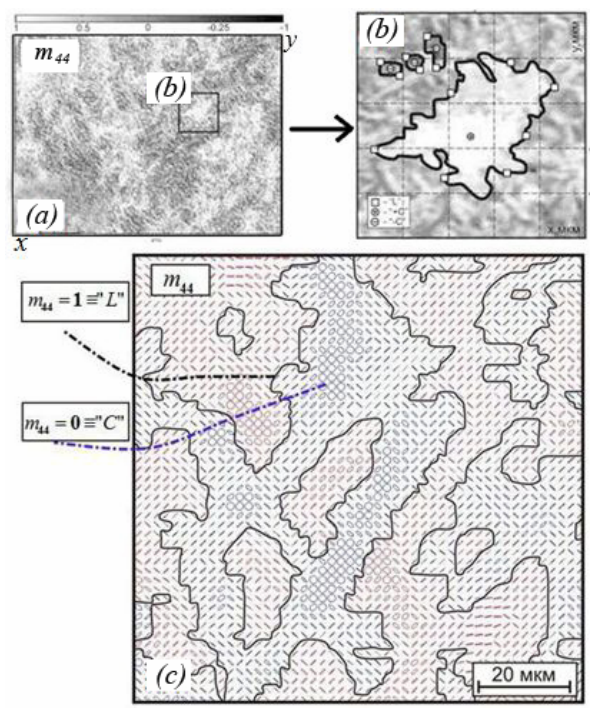

Fig. 1. Topographic structure of the phase Mueller-matrix pattern $(a, b)$ for skin derma and polarization-singular net (c) corresponding to the formed laser image.
The obtained data have confirmed adequacy of model conceptions about singular peculiarities in optical anisotropy of polycrystalline birefringent networks.

\section{Experimental investigations of characteristic values inherent to Mueller-matrix patterns of biological layers}

We performed investigations of the efficiency of using the one-point (singular) approach to the analysis of processes taking place under interaction of laser radiation with polycrystalline networks of biological liquid films. As an object under study, we chose effusion of operatively extracted human appendixes in catarrhal and gangrenous states. Represented in Fig. 2 are the coordinate samplings $(a, d)$ of characteristic values from the phase Mueller-matrix patterns $\left(m_{44}=0\right)$; distributions of the amounts $(N(x))$ for these values $(\mathrm{b}$, e); logarithmic dependences (c, f) for the power spectra of $(N(x))$ dependences typical for the cases of catarrhal (a to c) and gangrenous (d to f) states of appendixes.

Our analysis of these data indicates the growth of total amount of characteristic values $m_{44}=0$ for the polycrystalline network of the effusion film prepared from the gangrenous appendix. Fractal distributions of this sampling for the phase Mueller-matrix pattern corresponding to the effusion film for the case of human catarrhal appendix are transformed into statistical ones in the case of gangrenous appendix - the approximating curve for logarithmic dependences of power spectra corresponding to has no stable slope. Quantitatively, distributions of characteristic values $m_{44}=0$ are described with statistical, correlation and fractal parameters summarized in Table 2 .

Table 2. Statistical, correlation and fractal parameters of the sampling $m_{44}(m \times n)=0$ corresponding to Mueller-matrix patterns of the effusion film

\begin{tabular}{|c|c|c|}
\hline$M_{i=1-4}^{\varphi}$ & Norm & Adenocarcinoma \\
\hline$M_{1}^{\varphi}$ & $0,22 \pm 0,028$ & $0,36 \pm 0,042$ \\
\hline$M_{2}^{\varphi}$ & $0,23 \pm 0,03$ & $0,13 \pm 0,017$ \\
\hline$M_{3}^{\varphi}$ & $0,89 \pm 0,097$ & $2,17 \pm 0,27$ \\
\hline$M_{4}^{\varphi}$ & $0,77 \pm 0,081$ & $2,86 \pm 0,31$ \\
\hline$K_{2}^{\varphi}$ & $0,19 \pm 0,022$ & $0,31 \pm 0,033$ \\
\hline$K_{4}^{\varphi}$ & $0,89 \pm 0,0994$ & $2,64 \pm 0,27$ \\
\hline$W^{\varphi}$ & $0,27 \pm 0,033$ & $0,18 \pm 0,023$ \\
\hline
\end{tabular}

\section{(C) 2012, V. Lashkaryov Institute of Semiconductor Physics, National Academy of Sciences of Ukraine}



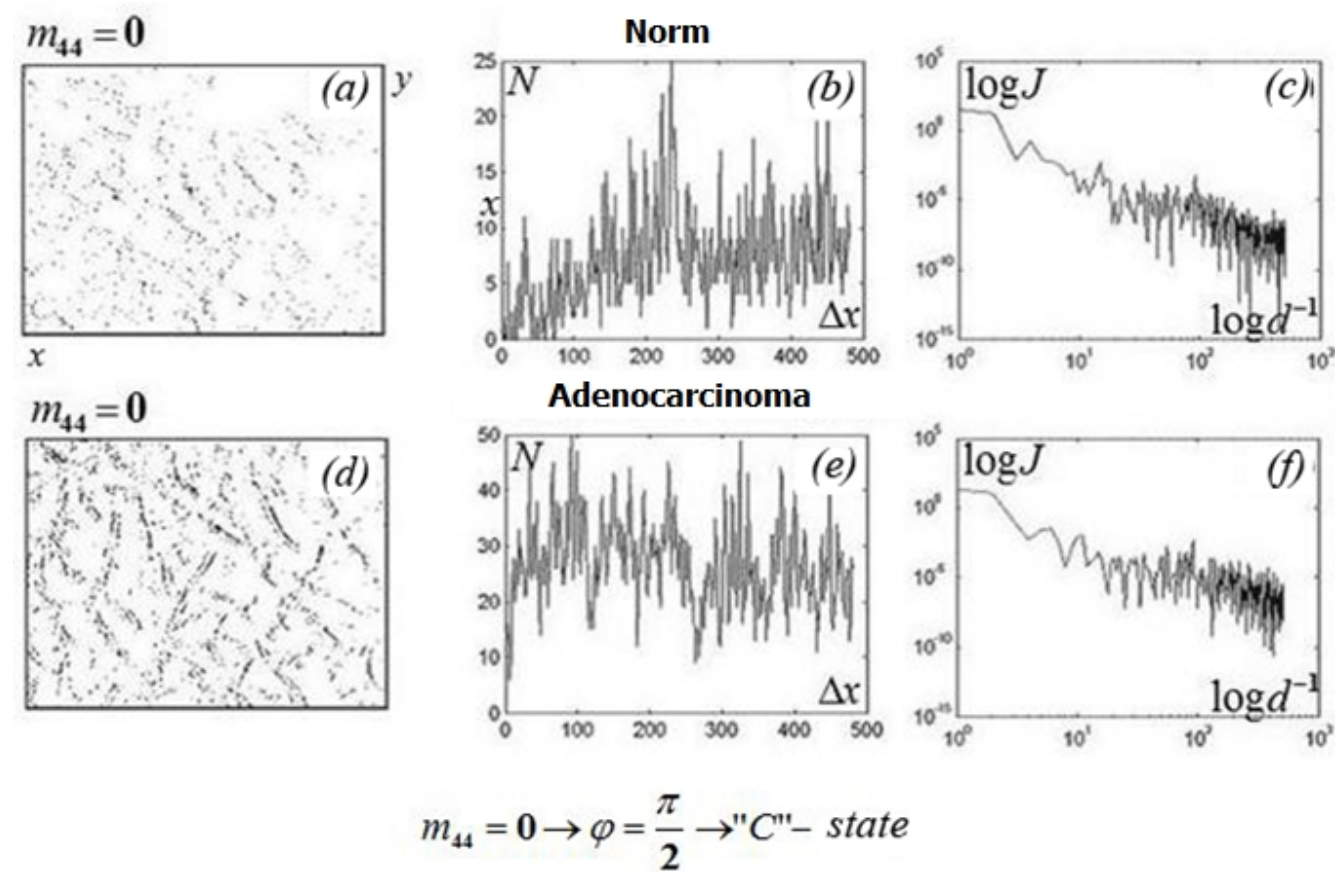

Fig. 2. Coordinate, quantitative and scale-self-similar structures of characteristic values found for Mueller-matrix patterns corresponding to films of appendix effusion for different physiological states. See explanations in the text.

These data show that the statistical moments of the third and fourth orders as well as excess of autocorrelation functions for distributions of amount of characteristic states in the phase Mueller-matrix pattern are diagnostically sensitive to changes of optical anisotropy in polycrystalline networks of effusion proteins with the specificity not less than $70 \%$ - the differences between them reach 2.4, 4.1 and 2.7 times, respectively.

\section{Polarization-correlation method for studying the Mueller-matrix patterns of biological layers}

Studied in this part of work was the possibility to use correlation juxtaposition of distributions for polarization-singular and characteristic states in the points of coordinate distributions for Mueller-matrix patterns instead of the one-point singular analysis. This approach was based on the method of measuring the coordinate distribution of the module for complex degree of mutual polarization (CDMP) related to the laser image of biological later with an arbitrary interval of discretization $\Delta r_{i}$

$W\left(r_{n+k}, r_{n}\right)=\frac{\left.\left(\left(I^{(0)}\left(r_{n+k}\right) I^{(0)}\left(r_{n}\right)\right)^{\frac{1}{2}}-\left(I^{(90)}\left(r_{n+k}\right) I^{(90)}\left(r_{n}\right)\right)\right)^{\frac{1}{2}}\right)^{2}}{I\left(r_{n+k}\right) I\left(r_{n}\right)}+$

$+\frac{4\left(I^{(0)}\left(r_{n+k}\right) I^{(90)}\left(r_{n+k}\right) I^{(0)}\left(r_{n}\right) I^{(90)}\left(r_{n}\right)\right)^{\frac{1}{2}} \cos \left(\delta_{n+k}\left(r_{n+k}\right)-\delta_{n}\left(r_{n}\right)\right)}{I\left(r_{n+k}\right) I\left(r_{n}\right)}$.
Here, $I^{(0)}\left(r_{i}\right) ; I^{(90)}\left(r_{i}\right)$ is the value of intensity measured for orientations of the transmission axes for the analyzer fixed at the angles $\Theta=0^{\circ}$ and $\Theta=90^{\circ}$.

The performed juxtaposition of polarizationcorrelation and singular approaches enabled us to to reveal that the averaged statistical size of correlation contour is corresponded with the halfwidth of autocorrelation function $G$ for the coordinate distribution of CDMP. To determine parameters of the correlation contour, we used the method of crosscorrelation analysis. The latter is based on the principle of determining the series of autocorrelation dependences and power spectra in two mutually orthogonal directions - $O x$ and $O y$.

$$
\begin{gathered}
W(x, y) \Rightarrow\left(\begin{array}{c}
G_{1}(\Delta x) ; \Delta x=1, \ldots, m \\
G_{y=n}(\Delta x) ; \Delta x=1, \ldots, m
\end{array}\right) \Rightarrow \bar{G}(\Delta x)=\sum_{i=1}^{n} G_{i}(\Delta x) ; \\
W(x, y) \Rightarrow\left(\begin{array}{c}
G_{1}(\Delta y) ; \Delta y=1, \ldots, n \\
G_{x=m}(\Delta y) ; \Delta y=1, \ldots, m n
\end{array}\right) \Rightarrow \bar{G}(\Delta y)=\sum_{j=1}^{m} G_{j}(\Delta y) .
\end{gathered}
$$

Using the relations (14), we introduced the coefficient of asymmetry for the correlation contour

$\chi=\frac{L_{x}}{L_{y}}=\frac{L(G(\Delta x)=0.5)}{L(G(\Delta y)=0.5)}$.

The developed cross-correlation approach have been illustrated using the data of computer modeling in Fig, 3 . 

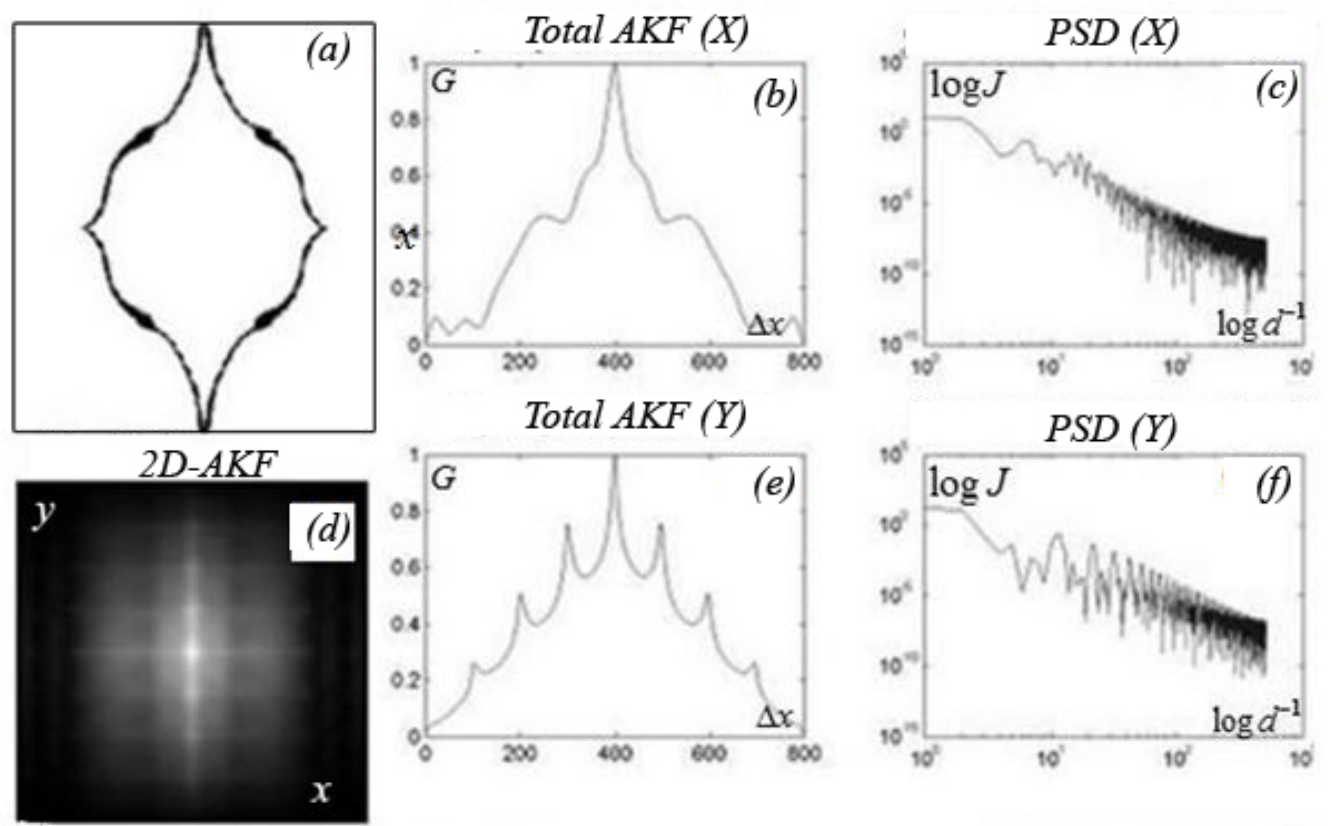

Fig. 3. Coordinate, autocorrelation and cross-correlation structures for CDMP distributions of circle-like birefringent cylinders.

As seen from the data obtained, in the case of orientation-phase modulation one can observe formation of a complex polarization-inhomogeneous field with an asymmetric two-dimensional autocorrelation function for the CDMP distribution. The developed method for polarization-correlation analysis was approved for differentiation of benign and malignant changes in biological tissues. Depicted in Fig. 4 are coordinate CDMP distributions for laser images of optically-thin histological sections taken from operatively extracted benign (left column) and malignant (right column) prostate tumors.

The obtained data illustrate asymmetry of correlation contours corresponding to coordinate distributions of CDMP modules for laser images typical for samples of both types. In the case of benign tumor, this contour is asymmetrical, which is caused by availability of predominant growth directions inherent to birefringent fibrils. In the case of malignant tumor, this asymmetry is practically two-fold reduced due to destruction of birefringent networks and respective azimuth symmetrization of coordinate changes in polarization states of laser images. Quantitatively, these differences in cross-correlation structures of twodimensional CDMP distributions are illustrated with the excess of autocorrelation functions and dispersion of logarithmic dependences for power spectra, which is summarized in Table 3.

Table 3. Cross-correlation parameters for distributions of CDMP modules.

\begin{tabular}{|c|c|c|}
\hline Parameters & Benign tumor & Malignant tumor \\
\hline$\chi$ & $2,05 \pm 0,28$ & $0,93 \pm 0,014$ \\
\hline$K_{4}$ & $1,82 \pm 0,29$ & $0,37 \pm 0,046$ \\
\hline$\Omega$ & $0,23 \pm 0,037$ & $0,48 \pm 0,057$ \\
\hline
\end{tabular}

It has been determined that the differences between correlation and spectral parameters oscillate between 2 to 5 times, which provides the specificity of this crosscorrelation method at the level $80 \%$.

As one application more, we considered the task to differentiate the causes of patient's death - ішемічна хвороба або гостра коронарна недостатність. Fig. 5 shows autocorrelation functions corresponding to coordinate distributions of a phase Mueller-matrix element of optically-thin histological sections taken from myocard tissue of the patien died as a consequence of ischemic heart disease (left column) and acute coronary insufficiency (right column).
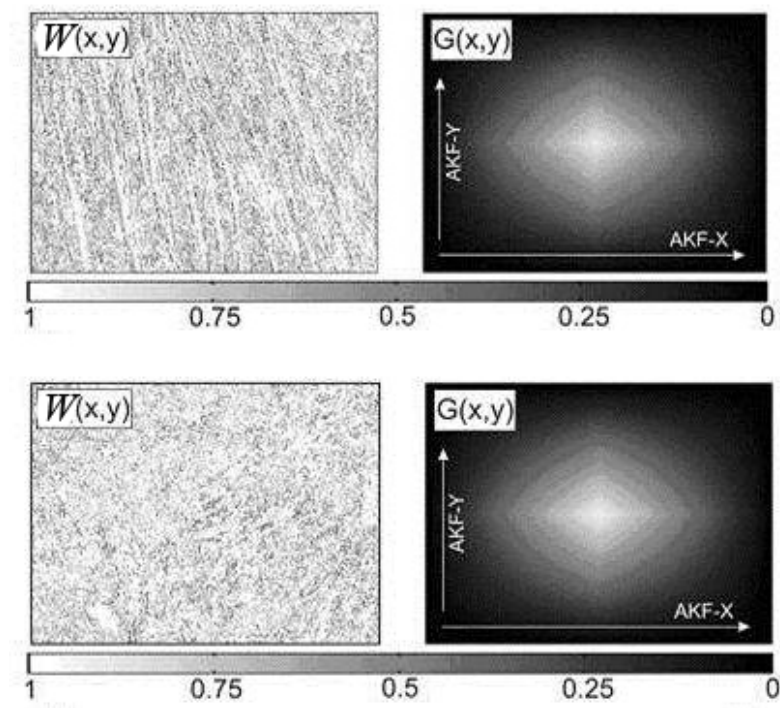

Fig. 4. Coordinate distributions for the CDMP module and their two-dimensional autocorrelation functions. 

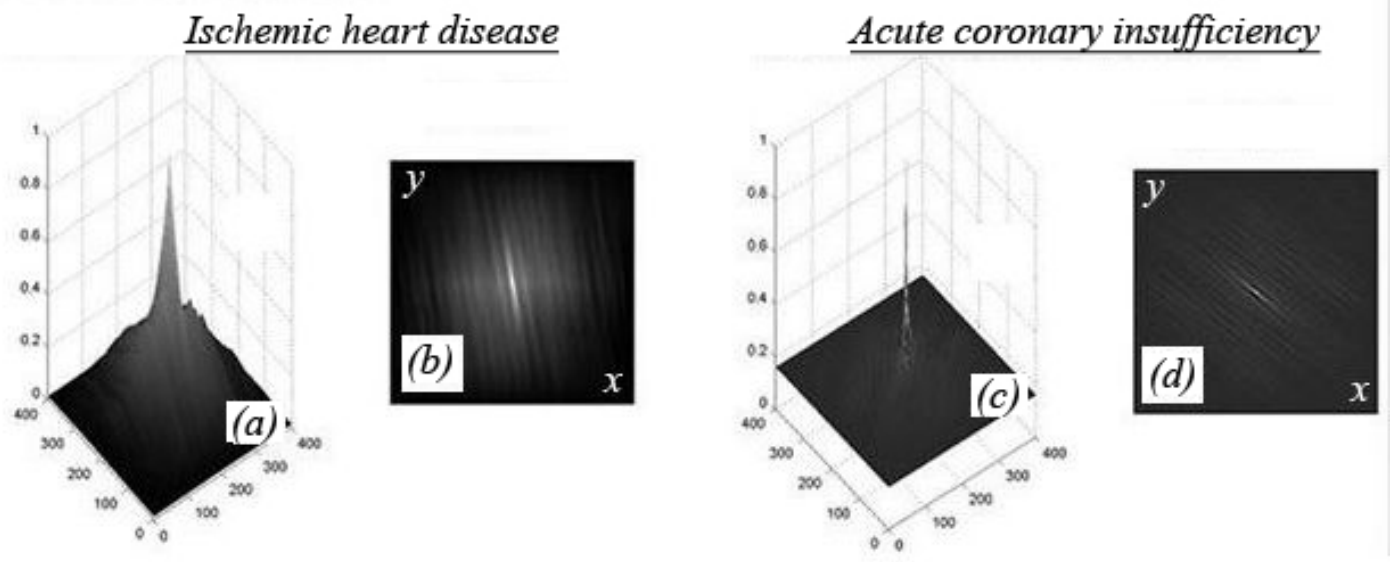

Fig. 5. Autocorrelation functions for coordinate distributions of the phase Mueller-matrix element typical for optically-thin histological sections of myocard tissue.

\section{Ischemic heart disease}

\section{Acute coronary insufficiency}
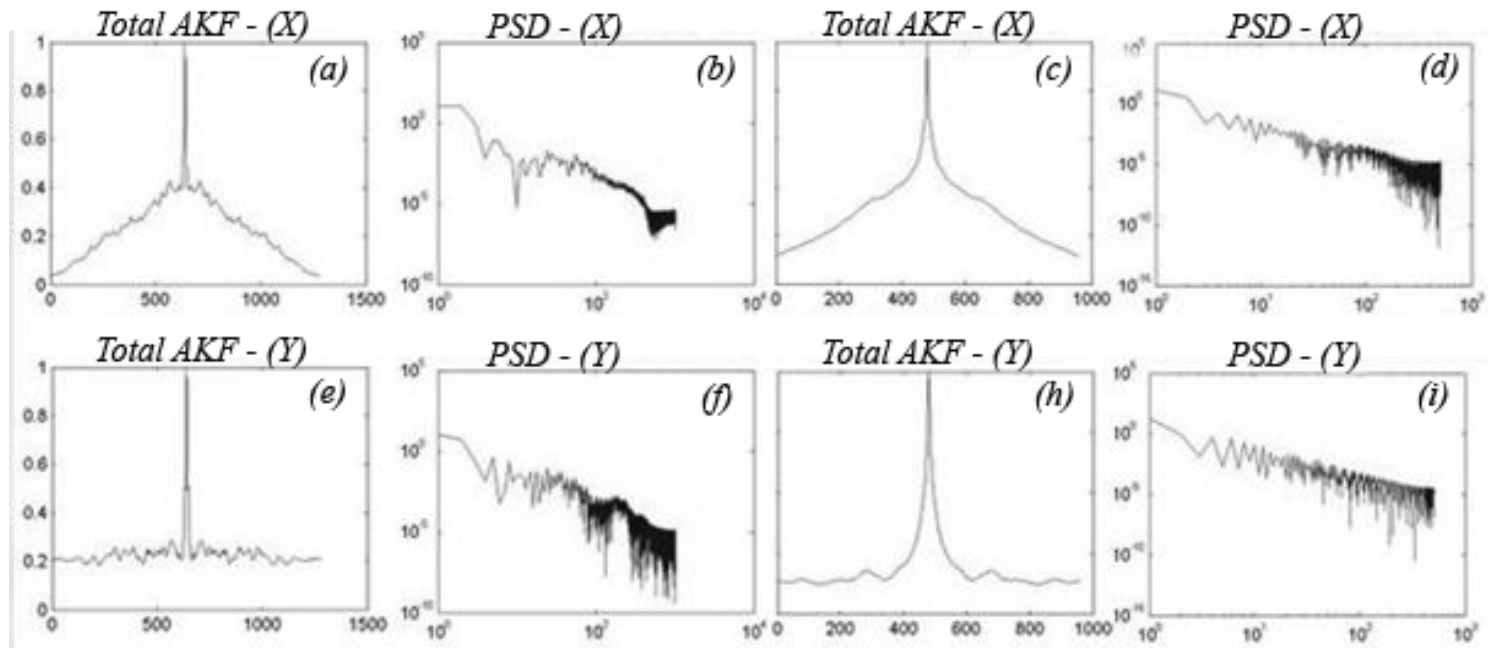

Fig. 6. Cross-correlation structures of Mueller-matrix patterns corresponding to histological sections of myocard tissue.

Depicted in Fig. 6 are the results of crosscorrelation analysis applied to Mueller-matrix patterns.

It was revealed that correlation and scale-selfsimilar structures of Mueller-matrix patterns for various myocard samples are different. In the case of ischemic disease (infarct), the coordinate distribution of phase matrix element is random owing to destruction and degradation of optical birefringence inherent to myosin fibers. In the case of acute coronary insufficiency, the respective Mueller-matrix pattern is scale-self-similar. From the quantitative viewpoint, the differences between cross-correlation structures of Mueller-matrix patterns are summarized in Table 4.

It has been found that the differences between correlation and spectral parameters oscillate from 2.5 up to 4.5 times, which provides the specificity of this crosscorrelation method at the level $87 \%$.
Table 4. Parameters of the cross-correlation analysis for phase Mueller-matrix patterns

\begin{tabular}{|c|c|c|}
\hline Parameters & $\begin{array}{c}\text { Ischemic heart } \\
\text { disease }\end{array}$ & $\begin{array}{c}\text { Acute coronary } \\
\text { insufficiency }\end{array}$ \\
\hline$\chi$ & $1,45 \pm 0,21$ & $4,93 \pm 0,59$ \\
\hline$K_{4}$ & $1,15 \pm 0,27$ & $5,17 \pm 0,96$ \\
\hline$\Omega$ & $0,44 \pm 0,067$ & $0,18 \pm 0,024$ \\
\hline
\end{tabular}

\section{Conclusions}

1. Within the frames of singular approach, we have analyzed scenarios of amplitude-phase modulation of laser images typical to polycrystalline networks of biological layers and, for the first time, on the 
base of introduced characteristic values of Muellermatrix patterns $\left(m_{i k}=0 ; \pm 1\right)$ ascertained interrelations between topographic distributions of optical axis directions and values of birefringence inherent to biological crystals and coordinate distributions for L- and C-points of their laser images.

2. Experimentally confirmed is availability and coordinate correlation of the topographical structure typical to distributions of characteristic values in Mueller-matrix patterns and nets of polarization singularities in laser images of biological tissues with different morphology of protein fibrilar networks.

3. Found is interrelation between changes in birefringence of fibrilar biological crystals and distributions of characteristic values in Muellermatrix patterns that are related with mechanisms of formation of $\mathrm{C}$-states in laser images. Being based on it, we have demonstrated for the first time the efficiency of statistical analysis applied to distributions of amounts of characteristic values in the phase Mueller-matrix patterns for birefringent protein networks when differentiating the opticallythin histological sections of biopsy taken from benign and malignant prostate tumors.

4. Ascertained are the main crystalo-optics mechanisms providing formation of nets of polarization singularities in laser images of polycrystalline networks observed in films of biological tissues with weak phase fluctuations. Coordinate distributions of L-states are caused by interaction of laser radiation with the substance of optically active proteins; C-states are formed owing to phase modulation of ordered needle-like protein crystals. Using this base, we developed the new method for Mueller-matrix singular diagnostics of changes in birefringence inherent to films of biological liquids and, for the first time, realized differentiation of various nosologies, namely: norm, inflammatory processes (osteoarthritis of joints, cholelithiasis, catarrhal and gangrenous appendicitis), oncological changes (prostate cancer).

5. For the first time, two-point polarizationcorrelation approach is applied to the analysis of the coordinate structure in Mueller-matrix patterns of the optically anisotropic component of biological tissues and liquids. It enabled us to develop the method for cross-correlation determination of parameters (averaged statistical sizes, asymmetry coefficient) describing the correlation contour that defines the topographic structure of characteristic values inherent to Mueller-matrix patterns of biological tissues and liquids.

6. Using the approximation of single light scattering, we have found, for the first time, interrelation between quantitative parameters (asymmetry coefficient for the correlation contour of the mutual anisotropy complex degree, correlation moment of the fourth order and spectral moment of the second order for distributions of the mutual anisotropy complex degree), which characterize asymmetry of the correlation contour for Mueller-matrix patterns and changes in birefringence of biological tissues. It enabled us to realize, for the first time, polarization-correlation differentiation of benign and malignant changes in histological sections of the prostate tumor.

\section{References}

1. Wang L.-H. Propagation of polarized light in birefringent turbid media: a Monte Carlo study / X. Wang, L. - H. Wang // J. Biomed. Opt. - 2002. Vol. 7. - P. 279-290.

2. Тучин В. В. Лазеры и волоконная техника в биомедицинских исследованиях / Тучин В. В. Саратов: Изд-тво Сарат. ун-та, 1998. - 384c.

3. Angelsky O. V. Polarization visualization and selection of biotissue image two-layer scattering medium / O. V. Angelsky, A. G. Ushenko, D. N. Burcovets, Yu. A. Ushenko // J. Biomed. Opt. 2005. - Vol. 10, No.1. - P. 014010.

4. Nye J. F. Dislocations in wave trains. / J. F.Nye, M. Berry // Proc. R. Soc. Lond. - 1974. - Vol. A 336. - P. 165-190.

5. Berry M. V. Umbilic points on Gaussian random surfaces / M. V. Berry, J. H. Hannay // J. Phys. A: Math. Gen. - 1977. - Vol. 10. - P. 1809-1821.

6. Soskin M. Topological networks of paraxial ellipse speckle-fields / M. Soskin, V. Denisenko, R. Egorov // J. Opt. A: Pure Appl. Opt. - 2004. - Vol. 6. - P. S281-S287.

7. Gori F. Beam coherence-polarization matrix / F. Gori, M. Santarsiero, S. Vicalvi, R. Borghi, G. Guattari // Pure Appl. Opt. - 1998. - Vol. 7. - P. 941-951.

8. Tervo J. Degree of coherence for electromagnetic fields / J. Tervo, T. Setala, A. Friberg. // Opt. Express. - 2003. - Vol. 11. - P. 1137-1143.

9. Ellis J. Complex degree of mutual polarization / J. Ellis and A. Dogariu // Opt. Lett. - 2004. - Vol. 29. - P. 536-538. 